\title{
Improved management in the diagnosis of gonorrhoea in women
}

\author{
A. E. JEPHCOTT AND S. RASHID \\ From the Public Health Laboratory, Northern General Hospital and the Special Treatment Clinic, \\ Royal Infirmary, Sheffield
}

SUMMARY In this paper an evaluation is made of the endeavours to improve diagnosis in women named as contacts of gonorrhoea. The problem was approached in three ways. (a) The number of sites sampled was increased. (b) The results of microscopical examination of smears made by clinical staff were regularly evaluated. (c) Cultural examinations by the supporting laboratory were dealt with more efficiently and speedily. It is concluded that the number of tests currently used to establish or exclude a diagnosis of gonorrhoea in women can safely be reduced to two, and that the methods currently in use allow a more cost-effective management of an increasing case load.

\section{Introduction}

Efficient diagnosis of gonorrhoea is essential to control the disease. Until recently it was usual to make three separate examinations of genital specimens before eliminating gonococcal infection in a female contact or in a suspected case. This can be a time-consuming and an unrewarding procedure. Recent advances in laboratory techniques have so improved diagnostic efficiency that it is doubtful if as many examinations are still necessary. Accordingly a review of the performance of the diagnostic service in Sheffield during the past eight years was made to establish the cost effectiveness and medical necessity of repeated examinations.

\section{Patients and methods}

The notes of all cases of gonorrhoea in women diagnosed during the first quarters of the years 197077 were reviewed to establish how the diagnoses had been reached and whether this had been at the first, second, or third examination. Cases in which the diagnosis was made at the third examination or later were reviewed in detail.

Routinely patients were examined on three occasions and samples were taken for microscopical examination and culture. The examinations were

Address for reprints: Dr A. E. Jephcott, Public Health Laboratory, Myrtle Road, Bristol

Received for publication 31 October 1977 made at the earliest opportunity, daily if possible. Initially, urethral and endocervical samples only were examined. Since early in 1974 rectal samples taken through a proctoscope have also been examined. From the same time, pharyngeal samples have been cultured selectively if examination of the first set of Gram-stained smears gave negative results or if there was a history of fellatio.

The methods of sampling for smear preparation, Gram staining, and microscopical examination were recommended by Morton (1977). All smears, both diagnostic and post-treatment, were read at the time of the patients' attendance. Until 1976 three followup sets of tests were routine. Thereafter two sets were used. All diagnostic smears were retained until the results of the corresponding culture specimens were available. In cases of disparate findings the smears were reassessed. This day-by-day appraisal of findings, together with periodic evaluation of smear and culture methods, was applied by medical and nursing staff during the eight-year study period.

The clinic and supporting laboratory are two miles apart. In the earlier years Stuart's transport medium was used to protect all specimens sent for culture. On reaching the laboratory they were plated on to a non-selective heated blood medium (McLeod et al., 1934). A selective medium (Phillips et al., 1972) was introduced late in 1970. Such media inhibit the growth of some gonococci (Reyn, 1973), so a selective medium and one that was nonselective were used in parallel for all samples submitted in transport medium after that date. Since 
1974 Amies' transport medium has been used in preference to Stuart's.

In 1974 samples obtained at the first examination were inoculated directly on to a Transgrow transportcum-culture medium. If second and third examinations were carried out Amies' transport medium was used (Jephcott et al., 1974). Jembec transport-cumculture medium replaced Transgrow for first examination samples in 1975 and has been in use ever since (Jephcott et al., 1976).

Before routine use all new media were evaluated by using specimens from men with urethral Gram stains showing typical Gram-negative intracellular diplococci.

Suspicious colonies were submitted to Gram stain and oxidase testing and were finally identified by sugar fermentation (Flynn and Waitkins, 1972). Since 1974 an immunofluorescent (IF) method has been used routinely (Jephcott, 1977). The biochemical method is still used if the results from the IF method are unsatisfactory and for all rectal and pharyngeal isolates.

\section{Findings}

The Table shows the cases of gonorrhoea diagnosed in women during the first quarters of each of the eight years 1970-77. The number diagnosed by microscopical examination has remained relatively constant. This has resulted in a decrease in the proportion so diagnosed. There has been both an absolute and a relative increase in the numbers diagnosed by cultural methods. This is depicted graphically in Fig. 1 which also shows changes in methods. Figures 2-4 show the cases diagnosed at the first, second, and third tests by microscopy and culture. Figure 5 shows the proportion of cases diagnosed in the first two tests.

Throughout the entire study period seven cases $(0.9 \%)$ were diagnosed at the third (or in one case the fourth) attempt. These cases are of special interest and the findings are germane to a detailed appraisal. They are therefore reported in detail.

PATIENTS REQUIRING THREE OR MORE TESTS Patient 1

This patient was seen on 6 January 1970 as her consort had a urethral discharge. She was asymptomatic, her last sexual contact being the day before. Smears and cultures were negative. She attended again on 12 January reporting another exposure on 10 January. Tests were again negative but a third set of tests, taken on 13 January, revealed a positive cervical culture. Her consort was diagnosed on 12 January. She might have been infected on 10 January and not 5 January. It is therefore

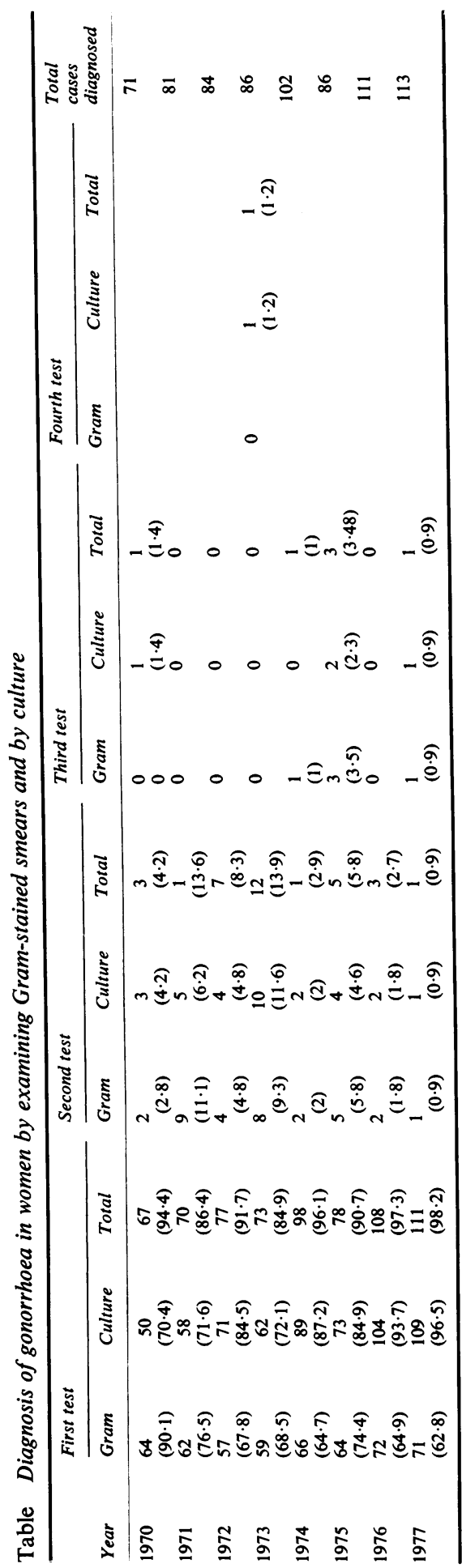




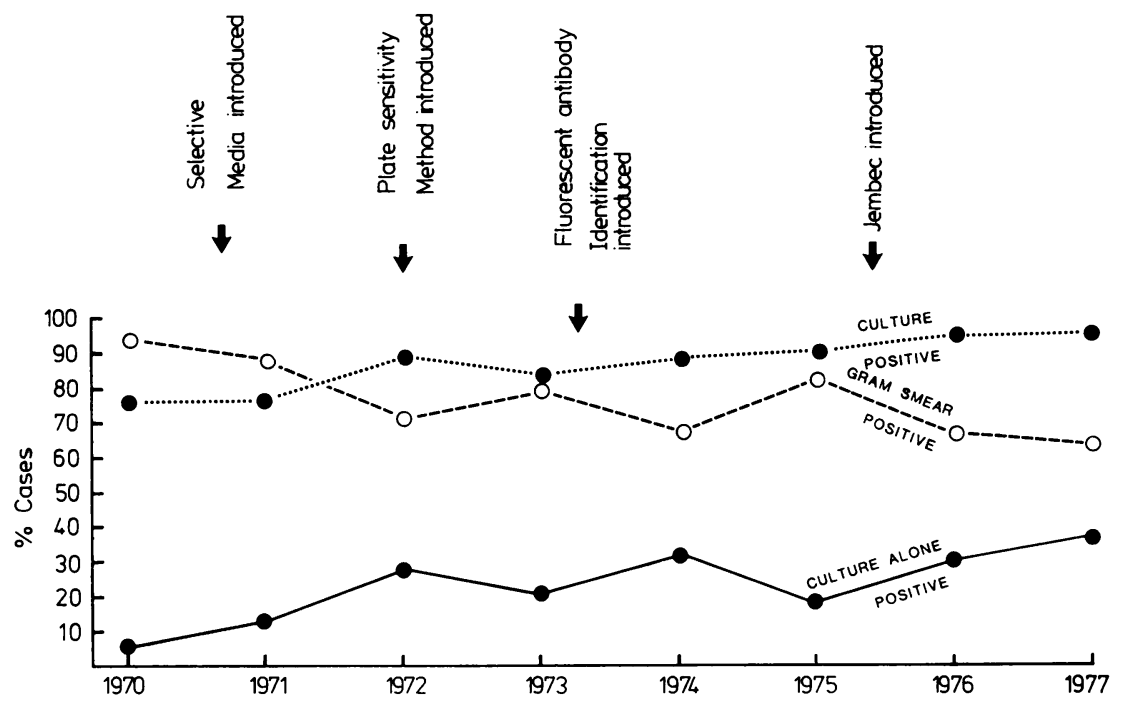

Fig. 1 Percentages of cases of gonorrhoea diagnosed by examination of smear or culture, January-March 1970-77

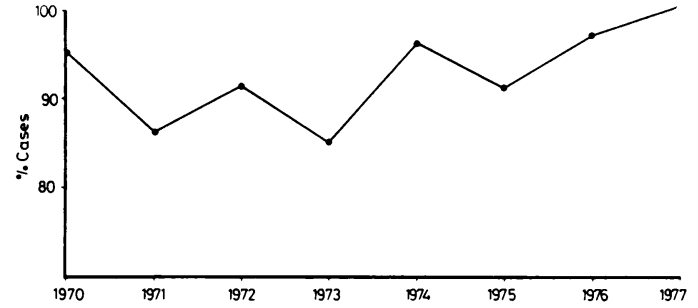

Fig. 2 Total number of women diagnosed in the first test

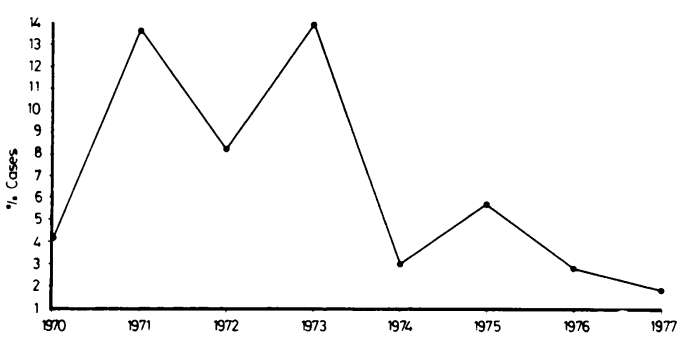

Fig. 3 Total number of women diagnosed in the second test

difficult to establish the number of examinations made after infection.

\section{Patient 2}

This patient was a prostitute. She first attended with a vaginal discharge on 14 March 1973, three days

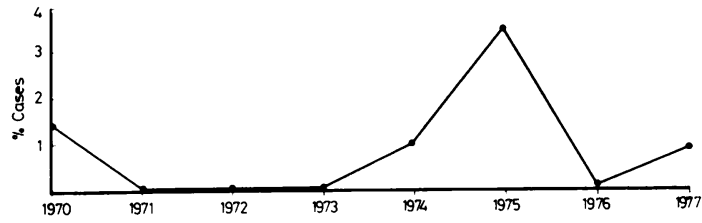

Fig. 4 Total number of women diagnosed in the third test

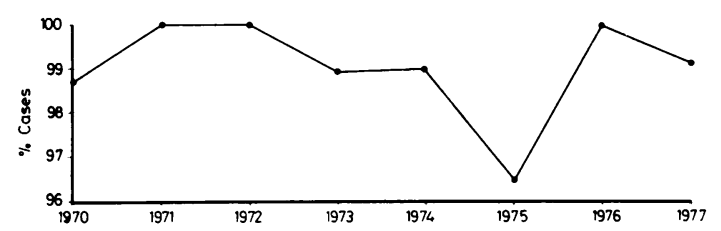

Fig. 5 Total number of women diagnosed with two tests

after her last sexual contact. She had had four different consorts during the previous month. The night before attending she had been given six antibiotic tablets (probably penicillin and analgesics) by her general practitioner. Three sets of tests on 14,15 , and 16 March gave negative results, but because symptoms persisted and treatment was inadequate a further set of tests was taken on 19 March. This time the cervical culture result was positive. Gonorrhoea was diagnosed in one of her consorts at his first attendance at the clinic on 
20 March. Inadequate oral antibiotic treatment could have masked her infection while failing to eradicate it, or, she might not have been infected until between the 16 and 19 March-the case notes are unhelpful on this point.

\section{Patient 3}

This patient was seen initially on 18 January 1974 as the asymptomatic consort of a man with gonorrhoea who had been diagnosed and treated three weeks before. Examinations made on 18 and 22 January gave negative results but a rectal smear examination made on 24 January proved positive. All other smears and cultures gave negative results. Rectal smears are often difficult to read, but this smear was reassessed after the negative culture result became known.

\section{Patient 4}

This patient first attended on 27 December 1974 with symptoms. Tests taken on 27 and 30 December gave negative results but a third set, taken on 6 January 1975, yielded positive results to smears and cultures. Last sexual contact was said to be five months previously, although the symptoms were of recent onset. The history may be considered questionable in this instance.

\section{Patient 5}

This patient was one consort of a man who was diagnosed as having gonorrhoea and was treated on 20 January 1975. She attended on 21 January 1975, 10 days after her last sexual contact. Her history was confirmed by the consort. Tests taken on 21 and 22 January gave negative results but a rectal smear was found to be positive in the third set of tests taken on 23 January. All other smears and cultures taken that day gave negative results. The man had had one other consort but she did not attend the clinic. Again the difficulty of reading rectal smears is evident.

\section{Patient 6}

This patient attended on 23 January 1975, one week after contact with a man who was found to be suffering from non-specific urethritis. His urethral smear and culture showed no gonococci and he gave no history of recent treatment with antibiotics. The patient was examined on 22 and 24 January 1975 with negative results, but on 31 January cervical smears and cultures both proved positive. The patient admitted to no other consort during the previous six months.

\section{Patient 7}

This patient first attended on 12 February 1977 as a contact of her husband who was diagnosed as having gonorrhoea and was treated on the same day. Her last marital contact was stated to be five days before and her husband confirmed this. Tests made on 12 and 14 February were negative, but both smears and cultures gave positive results at a third examination on 15 February. The patient denied any other contact. The husband had had a casual contact one week before attendance. It is possible that the first examinations were carried out during the wife's incubation period if the history is disregarded, but many other explanations are also possible in this instance.

\section{Discussion}

The number of examinations required to diagnose gonorrhoea in women has recently been discussed. Chipperfield and Catterall (1976) suggested that only two examinations were necessary. They did not use selective media or IF methods, however, and did not normally obtain rectal samples. Evans (1976) did use a selective medium but he did not use it in parallel with a non-selective one. He used IF identification but did not examine rectal specimens routinely. These two omissions are known to lead to missing between 3 and $5 \%$ of diagnoses (Reyn, 1973; Bhattacharyya and Jephcott, 1974). Thin et al. (1971) were among the first to use IF identification; they diagnosed $89 \%$ of their cases in two sets of tests.

There seems to be little doubt that the endeavours of supporting laboratories have effected improvements. This applies to isolation of gonococci and to the speed with which they can be identified by the IF method (Jephcott et al., 1974). Analysis of the figures in this study shows that the first samples provided $98 \%$ of the diagnoses. This was increased to over $99 \%$ of the total when two tests were done.

A detailed review of cases diagnosed at a third examination (or later) suggests that there are reasons for late diagnosis. For example, the patient's history may be misleading (as for Patients 4 and 6), treatment with antibiotics may be insufficient to effect cure but may make difficulties (as in Patient 2), and there are suggestions that findings made on examination of Gram-stained rectal material are liable to be unconfirmed (Patients 3 and 5).

It is of interest to note that although the aim was to secure the attendance of all named contacts as early as possible and to repeat tests as near to daily as possible, there was no obvious failure owing to the patient being examined too soon, but Patient 7 suggests that this may theoretically be a possibility. 


\section{Conclusion and recommendation}

It is concluded from this study that the methods currently in use in Sheffield warrant a reduction in the number of examinations for establishing or excluding the diagnosis of gonorrhoea in women. In the face of an ever-increasing case load we believe that the growing numbers can be efficiently managed by two sets of tests instead of the current three, provided that the following criteria are fulfilled:

(a) The sites sampled routinely include the rectum, and the pharynx when indicated.

(b) Gram-stained smear findings are continuously subjected to evaluation in terms of sampling, preparation, staining, and bacteriological findings.

(c) While a selective transport-cum-culture medium offers a speedy diagnosis, its use should be confined to the first examination. Second samples should be submitted for culture on both selective and non-selective media.

In addition the methods outlined offer improved management for individual patients and more objective economical use of personnel and materials, and are likely to prove more cost-effective than their predecessors. Furthermore, increased efficiency and speed of diagnosis reduces the number of infectious 'patient-days' in the community and so makes a worthwhile contribution to disease control.
We wish to thank Dr R. S. Morton for advice and encouragement during the preparation of this manuscript.

\section{References}

Bhattacharyya, M. N., and Jephcott, A. E. (1974). Diagnosis of gonorrhoea in women: Role of the rectal sample. British Journal of Venereal Diseases, 50, 109-112.

Chipperfield, E. J., and Catterall, R. D. (1976). Reappraisal of Gramstaining and cultural techniques for the diagnosis of gonorrhoea in women. British Journal of Venereal Diseases, 52, 36-39.

Evans, B. A. (1976). Detection of gonorrhoea in women. British Journal of Venereal Diseases, 52, 40-42.

Flynn, J., and Waitkins, S. A. (1972). A serum-free medium for testing fermentation reactions in Neisseria gonorrhoeae. Journal of Clinical Pathology, 25, 525-527.

Jephcott, A. E. (1977). Laboratory diagnosis of gonorrhoea. MD thesis, University of Sheffield.

Jephcott, A. E., Bhattacharyya, M. N., and Jackson, D. H. (1976). Improved transport and culture system for the rapid diagnosis of gonorrhoea. British Journal of Venereal Diseases, 52, 250-252.

Jephcott, A. E., Morton, R. S., and Turner, E. B. (1974). Use of transport-and-culture medium combined with immunofluorescence for the diagnosis of gonorrhoea. Lancet, 2, 1311-1313.

McLeod, J. W., Coates, J. C., Happold, F. C., Priestley, D. P., and Wheatley, B. (1934). Cultivation of the gonococcus as a method in the diagnosis of gonorrhoea, with special reference to the oxidase reaction and the value of air reinforced in its carbon dioxide content. Journal of Pathology and Bacteriology, 39, 221-231.

Morton, R. S. (1977). Major Problems in Dermatology: Gonorrhoea, volume 19, pp. 37-39. Saunders: London.

Phillips, I., Humphrey, D., Middleton, A., and Nicol, C. S. (1972). Diagnosis of gonorrhoea by culture on a selective medium containing vancomycin, colistin, nystatin, and trimethoprim (VCNT). British Journal of Venereal Diseases, 48, 287-292.

Reyn, A. (1973). Bakteriologisk undersøgelse for gonoré. Ugeskrift for Laeger, 135, 909-913.

Thin, R. N. T., Williams, I. A., and Nicol, C. S. (1971). Direct and delayed methods of immunofluorescent diagnosis of gonorrhoea in women. British Journal of Venereal Diseases, 47, 27-30. 